\title{
MVK mutations and associated clinical features in Italian patients affected with autoinflammatory disorders and recurrent fever
}

Andrea D’Osualdo ${ }^{1,2}$, Paolo Picco ${ }^{1}$, Francesco Caroli ${ }^{2}$, Marco Gattorno ${ }^{1}$, Raffaella Giacchino $^{3}$, Patrizia Fortini ${ }^{4}$, Fabrizia Corona ${ }^{5}$, Alberto Tommasini ${ }^{6}$, Giuseppe Salvi ${ }^{7}$, Fernando Specchia ${ }^{8}$, Laura Obici ${ }^{9}$, Antonella Meini ${ }^{10}$, Antonio Ricci ${ }^{11}$, Marco Seri ${ }^{12}$, Roberto Ravazzolo ${ }^{2,13}$, Alberto Martini ${ }^{1}$ and Isabella Ceccherini*,2

\footnotetext{
${ }^{1}$ Divisione Pediatria II Clinica Pediatrica, Genova, Italy; ${ }^{2}$ Laboratorio di Genetica Molecolare, Genova, Italy; ${ }^{3}$ Divisione Malattie Infettive, Genova, Italy; ${ }^{4}$ Laboratorio di Analisi Cliniche, Istituto Giannina Gaslini, Genova, Italy; ${ }^{5}$ Centro di Reumatologia Infantile, Clinica Pediatrica De Marchi, Università di Milano, Milano, Italy; ${ }^{6}$ Clinica Pediatrica IRCCS Burlo Garofolo, Trieste, Italy; ${ }^{7}$ Malattie Infettive Clinica Pediatrica IV, Azienda Ospedaliera Meyer, Firenze, Italy; ${ }^{8}$ Dipartimento di Pediatria, Università di Bologna, Bologna, Italy; ${ }^{9}$ Biotechnology Research Laboratory, IRCCS Policlinico San Matteo, Pavia, Italy; ${ }^{10}$ Clinica Pediatrica, Spedali Civili, Brescia, Italy; ${ }^{11}$ Dipartimento di Pediatria, IRCCS Policlinico San Matteo, Università di Pavia, Pavia, Italy; ${ }^{12}$ Laboratorio di Genetica Medica, Università di Bologna, Bologna, Italy; ${ }^{13}$ Dipartimento di Pediatria e CEBR, Università di Genova, Genova, Italy
}

Autosomal recessive autoinflammatory disorder caused by mutations of the mevalonate kinase gene $(M V K)$, leading to mild, incomplete MK enzyme deficiency (MKD), has been known so far as Hyper-lgD and periodic fever syndrome (HIDS) and regarded as mostly occurring in Northern Europe. Here we report the results of the molecular characterization of the first Italian series of patients affected with

autoinflammatory disorders and periodic fever. A total of 13 different mutations, scattered throughout the $M V K$ coding region, were identified in either homozygous or compound heterozygous state in 15 patients. The mutation leading to the V $377 \mathrm{I}$ amino-acid change, already described also in other series, resulted the most common with a frequency of $50 \%$ of all MKD alleles. Among the other mutations, eight had never been described before, including an interstitial deletion of 19 nucleotides in exon 2 . In addition to these nucleotide changes, private and polymorphic $M V K$ variants have been detected in the patients under analysis and checked also in a set of control individuals. Clinical features are reported for each of the 15 MKD patients, and life-threatening infections and systemic amyloidosis presented as unexpected MKDrelated complications. Our study demonstrates that MKD is a common cause of recurrent fever also in the Italian population, where it is associated with both a wide spectrum of previously unreported $M V K$ mutations and peculiar phenotypic features.

European Journal of Human Genetics (2005) 13, 314-320. doi:10.1038/sj.ejhg.5201323

Published online 10 November 2004

Keywords: periodic fever; Hyper-IgD syndrome; mevalonate kinase; MVK mutation screening; $M V K$ common variants; MKD mevalonate kinase deficiency

*Correspondence: $\mathrm{Dr}$ I Ceccherini, Laboratorio Genetica Molecolare, Istituto G Gaslini, L.go G Gaslini, 5, 16148 Genova, Italy. Tel: + 39010 5636800; Fax: + 390103779797 E-mail: isa.c@unige.it

Received 20 April 2004; revised 14 September 2004; accepted 15 September 2004
Introduction

A mild form of mevalonate kinase deficiency (MKD), previously known as either Hyper-IgD and periodic fever 
syndrome (HIDS) or Dutch-type periodic fever (MIM 260920), is an autosomal recessive autoinflammatory disorder characterized by recurrent attacks of apparently unprovoked fever associated with lymphadenopathy, abdominal pain, arthralgia and skin rash. ${ }^{1}$ It has an early onset, usually in infancy, and febrile crises recur at varying intervals. Fever flareups have an abrupt onset and last approximately 4-6 days; an attack is heralded by chills and patients frequently complain of malaise, headache, nausea and diarrhoea. ${ }^{2}$ Firstly described in 1984 , its previous HIDS name was derived from the constitutively elevated levels of serum IgD, usually associated with increased serum IgA concentrations. $^{3,4}$ This finding has been used as a diagnostic hallmark until it has become clear that IgD levels are not always increased. Since an impaired function of mevalonate kinase (MK), encoded on chromosome 12q24 by the MVK gene, represents a constant feature, the term MKD seems more appropriate. ${ }^{5,6}$ The profound deficiency of the MK enzyme causes a distinct syndrome called mevalonic aciduria (MA; MIM 251170), which is clinically characterized by severe mental retardation, ataxia, failure to thrive, myopathy and cataracts; recurrent fever attacks are present too. ${ }^{7}$ MK is an essential enzyme in the isoprenoid biosynthesis pathway, which produces several biomolecules involved in different cellular processes. ${ }^{8}$ Although the dysregulation of this biochemical pathway seems to play a pivotal role in the developing of fever, at present the pathogenetic mechanisms leading to autoinflammatory recurrent disease remain still poorly understood.

In all, 47 sequence variants have been described so far (http://fmf.igh.cnrs.fr/infevers/) throughout almost all coding regions of the $M V K$ gene..$^{9-11}$ The most frequently occurring mutation is the c.1129G $>$ A transition, which changes the valine at position 377 into an isoleucine (V377I). ${ }^{12}$ This mutation has been exclusively found in patients with a mild MKD presentation, thus providing evidence in favour of a limited effect on decreased enzymatic levels, as confirmed by a study of V377I carriers and MKD patients, which has demonstrated low penetrance of this $M V K$ common variant when in homozygous state in the Dutch population. ${ }^{13}$ At present few patients homozygous for V377I have been described, while most affected individuals are compound heterozygotes for this mutation, and a second mutation that may have also been identified in MA. Typical MA mutations are those causing complete abolition of any MK activity and therefore can be found in the milder forms of MKD only as a part of compound heterozygotes. In addition to missense and nonsense mutations, also short interstitial deletions have been reported in these mildly affected MKD patients. $^{12}$

Here, we report clinical features and underlying $M V K$ genotypes of the first Italian series of patients affected with a MKD resembling the previously called HIDS.

\section{Patients and methods}

Starting from April 2002, the Gaslini Pediatric Institute in Genova (Italy) has established a nationwide laboratory facility for the genetic diagnosis of autoinflammatory disorders associated with recurrent fever. All the patients enrolled until December 2003 fulfilled the following criteria: (i) periodic or recurrent fever flareups with unexplained clinical symptoms; (ii) at least two of the following symptoms during attacks: lymphadenopathy, gastrointestinal involvement (vomiting, diarrhoea, pain), skin lesions (erythematous macules and papules), arthralgia, splenomegaly. Informed consent from the patients' parents was obtained in each case.

Molecular testing was performed in DNA extracted from peripheral blood lymphocytes by standard methods. The 10 coding exons (from 2 to 11) of the MVK gene (see Houten et $a l^{11}$ ) were analysed by means of denaturing high-performance liquid chromatography (DHPLC). Primers for PCR amplifications, amplicon lengths and PCR conditions are listed in Table 1 . All the PCR reactions were performed in a total volume of $25 \mu \mathrm{l}$ and run with an initial $12 \mathrm{~min}$ of denaturation at $95^{\circ} \mathrm{C}$, followed by 30 cycles at $95^{\circ} \mathrm{C}$ for $30^{\prime \prime}, T_{\text {ann }}$ reported in Table 1 for each amplicon for $30^{\prime \prime}$ and $72^{\circ} \mathrm{C}$ for $45^{\prime \prime}$, with a final extension at $72^{\circ} \mathrm{C}$ for $7 \mathrm{~min}$. Exons with altered elution profiles in DHPLC assay were directly sequenced with the appropriate primers.

To detect homozygous mutations, for each patient and each exon under study amplification products were mixed with an equal amount of those obtained from a control individual known to carry no variant alleles at the MVK locus, denatured, reannealed and analysed for heteroduplexes. DNA analysis of each exon was also performed in 50 control Italian individuals.

IgD plasma levels were estimated by standard radial immunodiffusion (LC Partigen kit, Dade Behring Manburg, Germany).

\section{Results}

A series of 136 individuals (65 males and 71 females) affected with recurrent fever of unknown origin was collected. Following analysis of the MVK gene, 15 patients (nine males and six females) were demonstrated to carry mutations in either homozygous or compound heterozygous state. Clinical and genetic characterization of these patients is reported in Table 2 .

In all, 13 patients were of Italian origin, in addition to one Polish and one Albanian. There was neither consanguinity between parents nor additional affected members in any of the 15 families. The febrile attacks appeared within 1-24 months of age and 11/15 patients developed the disease before ageing 1 year. Fever spikes lasted nearly 5 days and then resolved completely, all patients being 
Table 1 Primer sequences designed for the amplification of the 10 coding exons of the MVK gene

\begin{tabular}{|c|c|c|c|c|c|}
\hline Exon & Primers sequence $5^{\prime} \rightarrow 3^{\prime}$ & Position of the forward primer & Amplicon [bp] & T ann. $\left[{ }^{\circ} \mathrm{C}\right]$ & $\mathrm{Mg}^{++}[\mathrm{mM}]$ \\
\hline 4 & $\begin{array}{l}\text { F:CTAGTCGATTTTCTGTGTTCTGTTG } \\
\text { R:ACATGATGAGGACAGCCAATGG }\end{array}$ & F:c.227-85C & 362 & 57 & 2 \\
\hline 5 & $\begin{array}{l}\text { F:GGACCAGATGCTTGGAGTCAG } \\
\text { R:CAGTGAGAGAAGCCACGTCC }\end{array}$ & F:c.372-102G & 327 & 60 & 2 \\
\hline 6 & $\begin{array}{l}\text { F:GTGGACTTGTTCTTTCTGAGCTC } \\
\text { R:CCAGAATCCATTGATTCGATTCC }\end{array}$ & F:c.528-131G & 348 & 57 & 1.5 \\
\hline 7 & $\begin{array}{l}\text { F:CTGCACCTITTGTAACTGAAGC } \\
\text { R:TTGCAGCTAGTGCACTGTGAG }\end{array}$ & F:c.632-155C & 349 & 55 & 2 \\
\hline 9 & $\begin{array}{l}\text { F:CAGGAAAATGACAGTGGTCG } \\
\text { R:ATTGCAGAGCCACTGCCTTG }\end{array}$ & F:c.769-206C & 392 & 62 & 2 \\
\hline 10 & $\begin{array}{l}\text { F:GTGGGCATAGGACCTTGGCC } \\
\text { R:GGAATTCTCCAGGTGGACCC }\end{array}$ & F:c.886-62G & 284 & 57 & 2 \\
\hline 11 & $\begin{array}{l}\text { F:ATGATGAGCTTCTCCCACGG } \\
\text { R:ATCCAGAAAGGGGCATCTGG }\end{array}$ & F:c. $1040-62 A$ & 261 & 62 & 2 \\
\hline
\end{tabular}

asymptomatic between episodes. The recurrence was nearly monthly, though more frequent in some cases.

Cervical lymph-node enlargement was a constant feature in our series (15/15 patients), so evident in three cases to change their facial shape. Severe mesentheric lymph-nodes and thickened ileal wall was found in some patients (\#1, \#7 and \#11) who underwent echo-scan examination during an acute attack. Transient splenomegaly was reported too (seven patients), while in one patient (\#2) spleen enlargement was caused by associated $\beta$ thalassemia. Abdominal pain occurred in all patients studied during the acute phase, 12 patients showing also short-term diarrhoea attacks; no patient showed failure to thrive and/or laboratory sign of malnutrition. Skin rash with a maculopapular or urticarioid appearance was present in 7/15 patients. All patients suffered from arthralgia and/or myalgia and poor general conditions during the acute phase.

IgD plasma level was found increased or minimally increased $(>100 \mathrm{UI} / \mathrm{ml})$ in only $10 / 15$ patients, five of whom showed also increased IgA levels $(>400 \mathrm{mg} / \mathrm{dl})$, while for one of them data were not available (Table 2). Two cases (\#1 and \#8), reported in detail below, showed a remarkable increase of only IgA, a condition already reported in $\mathrm{MKD}^{14}$ and, finally, in three patients both IgD and IgA levels resulted in the normal range (Table 2).

Notably, two patients developed severe infections. One patient (\#1), already diagnosed and reported as HIDS in the past, ${ }^{15}$ developed presumably a lethal meningococcal sepsis. He suffered from monthly fever attacks, responsive to NSAID and steroid treatments, usually associated with abdominal pain. ${ }^{16}$ At the age of 6 , he developed a longterm fever attack (10 days) associated with severe malaise, headache, meningismus and widespread petecchiae; when the patient was admitted to our Institute, he was already deceased and the diagnosis of meningococcal sepsis was formulated upon historical grounds. Another patient (\#8) was admitted to our Institute with unconfirmed suspicion of primary immunodeficiency since he was suffering from recurrent pneumonia and he had developed one episode of purulent meningitis; at admission his general conditions were good and the diagnosis was addressed by the uncommonly increased IgA plasma concentrations $(905 \mathrm{mg} / \mathrm{dl}) .{ }^{14}$ It is worth noting that in both patients \#1 and \#8 the evaluation of the immunological function (serum immunoglobulin, lymphocyte subpopulations, complement cascade, immunological response to tetanus and poliovirus vaccination, oxidative metabolism of granulocyte) was normal.

One patient (\#12), described in detail elsewhere, ${ }^{17}$ developed systemic amyloidosis associated with nephrotic syndrome, a condition already reported once in a patient affected by HIDS. ${ }^{18}$

Another patient (\#6) affected by monthly recurrent fever attacks associated with severe abdominal pain was found 


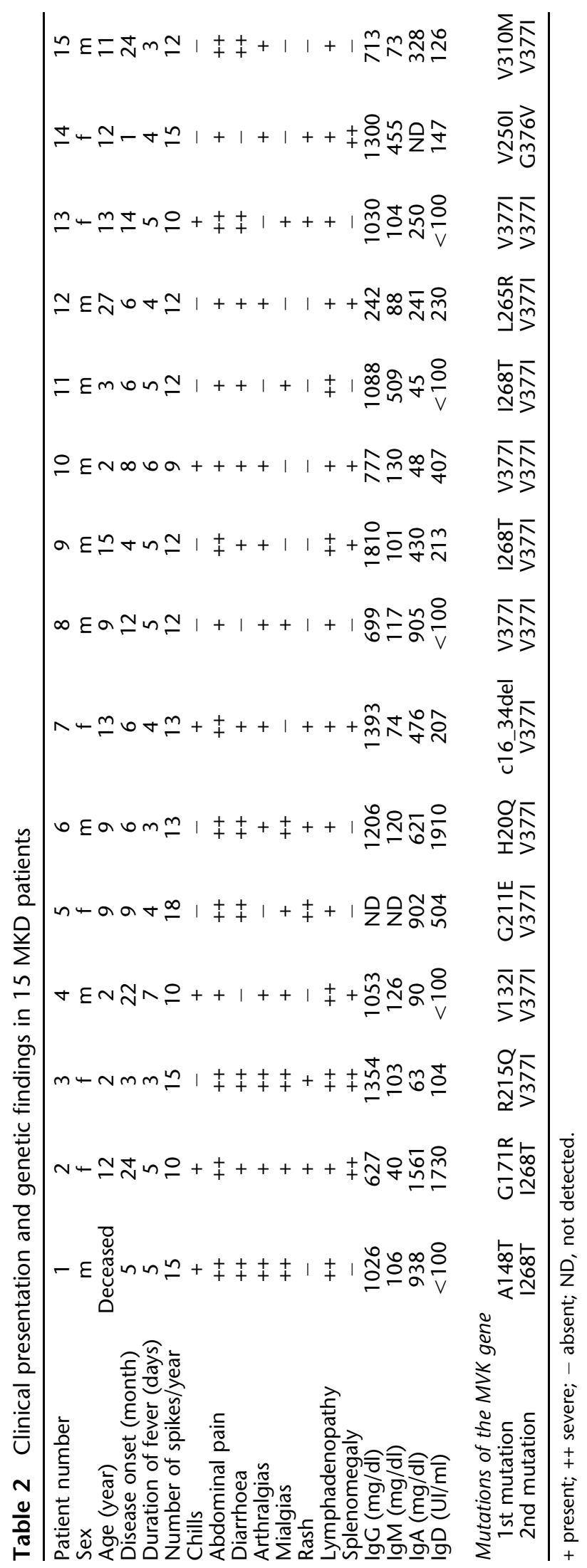

Table 3 MVK mutations never reported before

\begin{tabular}{lrcc}
\hline Patient & Exon & Mutant allele & Coding effect \\
\hline 7 & 2 & c.16_34del & frameshift \\
6 & 2 & c.60T $>$ A & H20Q \\
4 & 5 & c.394G $>$ A & V132I \\
2 & 5 & c.511G $>$ A & G171R \\
5 & 7 & c.632G $>$ A & G211E \\
14 & 8 & c.748G $>$ A & V250I \\
12 & 9 & c.794T $>$ G & L265R \\
14 & 11 & c. $1127 \mathrm{G}>\mathrm{T}$ & G376V \\
\hline
\end{tabular}

elsewhere to have a $\mathrm{R} 92 \mathrm{Q}$ mutation of the TNFRSF1A gene, known to display low penetrant association with the TRAPS phenotype. ${ }^{19}$ He had increased IgD level (1910 UI/ $\mathrm{ml}$ ) and $M V K$ gene analysis allowed the diagnosis of MKD.

Mutations identified in coding exons of the MVK gene, and corresponding amino-acidic changes induced in the MK protein, are reported in Table 2 for all 15 genetically diagnosed MKD patients. A total of 13 different mutations, scattered throughout the MVK coding region except for exons 3, 4 and 6, were identified. Among these 13 mutations, substitution of adenine for guanine at position 1129 (c.1129G >A), leading to a change from valine to isoleucine at codon 377 (V377I), was the most frequent one, being present in homozygous state in three patients and in heterozygous state in nine patients, with a final frequency of $15 / 30$ ( $50 \%$ of the 'MKD' chromosomes). The remaining mutations were all found in compound heterozygous state in patients, but not in 50 control individuals. Nonsense mutations were not found. Three mutations (A148T, R215Q and I268T) had already been reported in association with the MKD phenotype, ${ }^{12}$ while the c.928G $>$ A (V310M) mutation had previously been identified in one MA patient in homozygous state. ${ }^{20}$ The remaining eight mutations had never been described previously (Table 3 ). It is worth noting that three of them, H20Q, G211E and L265R, found in patients \#6, \#5 and \#12, respectively, affect codons which have already been involved in different MKD, both the previously called HIDS and MA, associated nucleotide and amino-acidic changes (H20N or H20P, G211A and L265P). Among the other five still unreported mutations, patient \#7 showed a deletion spanning 19 nucleotides of exon 2 (c.16_34del) and leading to a translation frameshift.

DNA of nine patients' parents (both parents of patients \#3, \#6, \#11, \#13 and mother of patient \#12) were available and in each case we confirmed the inherited occurrence of the mutations shown by the respective affected sons/ daughters.

During mutational analysis of the MVK gene, we also found different exonic and intronic polymorphic variants present both in the 136 patients affected with periodic fever and in 50 unaffected individuals (Table 4). In addition to two single-nucleotide polymorphisms (SNPs) already 
Table 4 Nucleotide variants of the MVK gene and their relative frequencies in 136 patients affected with recurrent fever and in 50 control individuals

\begin{tabular}{|c|c|c|c|c|c|}
\hline Exon/Intron & Position & Coding effect & Patients $^{\mathrm{a}}$ (no. 136) & Controls $^{\mathrm{a}}$ (no. 50) & P-value \\
\hline 5 & c. $405 \mathrm{G}>\mathrm{A}$ & S135S & 0.051 & 0.010 & 0.0714 \\
\hline 10 & c. $924 C>T$ & L308L & 0.004 & - & 0.5437 \\
\hline 11 & c. $1067 C>T$ & T356M & 0.004 & - & 0.5437 \\
\hline 11 & c. $1129 \mathrm{G}>\mathrm{A}$ & V377I & 0.059 & 0.020 & 0.1218 \\
\hline IVS2 & c. $79-62 \mathrm{G}>\mathrm{A}$ & - & 0.004 & - & 0.5437 \\
\hline IVS4 & c. $371+8 \mathrm{C}>\mathrm{T}$ & - & 0.096 & 0.090 & 0.8700 \\
\hline IVS6 & c. $632-18 A>C$ & - & 0.254 & 0.130 & 0.0107 \\
\hline IVS8 & c. $769-38 \mathrm{C}>\mathrm{T}$ & - & 0.158 & 0.080 & 0.0522 \\
\hline IVS8 & c. $769-7 \mathrm{~T}>\mathrm{G}$ & - & 0.004 & 0.010 & 0.4596 \\
\hline IVS9 & c. $885+24 G>A$ & - & 0.158 & 0.080 & 0.0522 \\
\hline $3^{\prime}$ UTR & c.1191+11C > T & - & 0.004 & - & 0.5437 \\
\hline
\end{tabular}

${ }^{\mathrm{a}}$ For each variant, the frequency of the least frequent allele is reported.

described (S52N and D170D), ${ }^{10}$ a c.405G $>$ A transition within exon 5 , resulting in a silent change (S135S), was found with similar frequencies in both patient and control chromosomes. Moreover, five private nucleotide variants were detected in heterozygous state in five patients, but neither in our control series nor in any other patient. This is the case of two synonymous changes (I260I and L308L), of a T356 M missense variant and of two intronic nucleotide substitutions (c.79-62G $>A$ and c. $1191+11 \mathrm{C}>\mathrm{T}$ ). Finally, another of these latter patients showed a single V377I mutant allele in the absence of any other MVK nucleotide change, and two carriers of the same allele were found in 50 control individuals.

All the remaining intronic variants have been found with a similar distribution in both patients and controls (Table 4), with the exception of the variant allele of the SNP c.632-18A $>$ G, which has revealed a frequency of 0.254 in the patients set and of 0.13 in the controls set $(P=0.0107)$. Finally, alleles of transitions c.769-38C $>\mathrm{T}$ and c. $885+24 \mathrm{G}>\mathrm{A}$ have resulted in complete linkage disequilibrium with each other.

\section{Discussion}

In this study, we focus on genetic findings and clinical features of the first Italian series of patients characterized by autoinflammatory disorders and recurrent fever associated with MKD, a condition known so far as HIDS. This latter definition does not comply any longer with this disease, because of the increasing evidences of patients showing normal IgD levels, as confirmed by the finding of normal or minimally elevated serum IgD in nearly half (seven out of 15) of the MKD patients reported here. Consequently, IgD level cannot be considered a sensitive marker for clinical MKD diagnosis.
The HIDS/MKD condition seems to be the most common cause of periodic fever in Northern Europe, and in the Netherlands a founder gene effect has recently been reported. ${ }^{21}$ Conversely, no epidemiological data are available in the Mediterranean basin, where this disease has been thought to be uncommon. The setting of a nationwide facility for the diagnosis of autoinflammatory disorders has allowed us to disclose that MKD represents a common cause of inherited periodic fever syndrome in Italy, as confirmed by the detection of MVK mutations in 15 out of a total of 136 patients enrolled in the study (11\%).

The early onset of the disease, the presence of abdominal involvement and increased IgD and IgA plasma concentrations were common features shared by the majority of our 15 diagnosed patients. Nonetheless, some patients showed relevant and previously unreported clinical features. Two out of 15 patients developed severe infectious complications, although their immunological function was apparently normal. This finding, in keeping with a recent report of MA in premature siblings with recurrent septicaemia, ${ }^{22}$ should be carefully considered in the current management of MKD patients to timely recognize infectious diseases, which might be misdiagnosed as periodic fever spikes and not promptly treated with antibiotic therapy. Moreover, as reported in detail elsewhere, ${ }^{17}$ our patient \#12 confirms a previous evidence that AA amyloidosis may also occur in association with the MKD syndrome. ${ }^{18}$ The finding of lifethreatening infectious and systemic amyloidosis imposes attention in the clinical management of these MKD patients and deserves further investigations to search for underlying molecular mechanisms.

Among our series of 136 selected patients with recurrent fever syndromes, a total of 15 patients was found to carry two mutant alleles of the MVK gene. In all, 15 of the 30 
associated mutations thus detected were V377I alleles, which has therefore resulted the most common genetic defect underlying MKD development in our country. Based on a frequency of 0.5 , the expected ratio of 1:2:1 between homozygotes V377I/V377I, compound heterozygotes V377I/nonV377I and patients carrying two nonV377I mutations does not differ significantly from the observed ratio of $3: 9: 3 \quad(P=0.74)$, thus confirming that mutant genotypes found in our patients series are in HardyWeinberg equilibrium. This finding differs from that previously reported for the same mutation in the Dutch population where the MVK genotypes are not in HardyWeinberg equilibrium, with respect to the V377I allele. ${ }^{13}$ Since the HIDS frequency has also been estimated lower than expected, these observations have suggested the authors to propose a low penetrance of the V377I homozygous genotype. ${ }^{13}$ Such a discrepancy between Italian and Dutch V377I allele distribution might be reconciled considering either the wide and not conservative criteria applied to the establishment of our patients series and/or the possible effect of other gene variants, different in the two populations, able to modify the phenotype of the V377I carriers. In addition to the V377I mutation, 12 other nucleotide changes were identified, all in compound heterozygous state in the $15 \mathrm{MKD}$ patients. Among these, mutations A148T, R215Q, I268T and mutation V310M had already been reported in association with the HIDS and the MA phenotypes, respectively. The remaining eight mutations had never been described previously, however, both their occurrence as part of a compound heterozygous genotype and some functional considerations support a role in inducing decreased level of MK enzymatic level for most of them. In particular, mutations H20Q, G211E and L265R are likely causative, affecting codons already involved in different MKDassociated changes (H20N/H20P, G211A and L265P), while a fourth, unreported mutation (c.16_34del) has a clear impairing effect of translation frameshift. The other four novel $M V K$ mutations reported here for the first time, V132I, G171R, V250I and G376V, affect amino-acidic residues which are either conserved in other mammalian species and/or located in close vicinity of stretches of highly conserved residues. ${ }^{23}$

Other mutant $M V K$ alleles were found in heterozygous state in six out of the 136 selected patients. With the exception of the case of the common V377I allele, the other five alleles are private variants represented by two synonymous changes (I260I and L308L), a T356 M missense change and two nucleotide substitutions (c.79$62 \mathrm{G}>\mathrm{A}$ and $\mathrm{c} .1191+11 \mathrm{C}>\mathrm{T}$ ) in intron 2 and in the $3^{\prime}$ UTR, respectively. Absence of all these latter changes in control chromosomes supports their possible effect on some either exonic or intronic splicing sites and a likely role in the respective patients in modifying, rather than causing, the disease phenotype, which might be sustained instead by defects in gene(s) to be yet identified. The same might be true for the patient carrying a single copy of the V377I allele in the absence of any other MVK defect, though in this case the presence of the heterozygous mutation, common also in control individuals, in association with a recurrent fever phenotype might be coincidental.

Data collected during our molecular study have also been used to test the possible association of the autoinflammatory with recurrent fever phenotype with MVK SNPs (Table 4). In particular, among the 10 common variants identified mainly in the noncoding region of the gene, alleles of SNP c.632-18A $>\mathrm{G}$ have resulted differentially distributed between the 136 patients and the 50 controls. This suggests either a direct effect on the intron 6 splicing and transcript maturation or a linkage disequilibrium with another noncoding variant, which would induce itself a mild decrease in the MK level, thus predisposing to autoinflammatory disorder(s), a hypothesis in need of further investigations.

In conclusion, our study shows that MKD is a cause of recurrent genetic fever also in the Italian population where a large spectrum of previously unreported MVK mutations and SNPs variants has been detected in many patients, and checked also in control individuals. Moreover, while most MKD conditions are generally considered benign, three out of 15 of our patients showed life-threatening complications such as recurrent severe infections and amyloidosis.

\section{Acknowledgements}

We thank Marco Di Duca and Giuseppe Santamaria for their excellent technical help. The financial support of the Italian Ministry of Health, the Italian Ministry of University (FIRB grant to RR) and the University of Genova is gratefully acknowledged.

\section{References}

1 Prietsch V, Mayatepek E, Krastel $\mathrm{H}$ et al: Mevalonate kinase deficiency: enlarging the clinical and biochemical spectrum. Pediatrics 2003; 111: 258-261.

2 Drenth JP, Haagsma CJ, van der Meer JW, International Hyper-IgD Study Group: Hyperimmunoglobulinemia D and periodic fever syndrome. The clinical spectrum in a series of 50 patients. Medicine 1994; 73: 133-144.

3 van der Meer JW, Vossen JM, Radl J et al: Hyperimmunoglobulinaemia D and periodic fever: a new syndrome. Lancet 1984; 1: 1087-1090.

4 Klasen IS, Goertz JH, van der Wiel GA, Weemaes CM, van der Meer JW, Drenth JP: Hyper-immunoglobulin A in the hyperimmunoglobulinemia D syndrome. Clin Diagn Lab Immunol 2001; 8: $58-61$.

5 Drenth JP, Cuisset L, Grateau G et al: Mutations in the gene encoding mevalonate kinase cause hyper-IgD and periodic fever syndrome. Nat Genet 1999; 22: 178-181.

6 Houten SM, Kuis W, Duran M et al: Mutations in MVK, encoding mevalonate kinase, cause hyperimmunoglobulinaemia D and periodic fever syndrome. Nat Genet 1999; 22: 175-177.

7 Hoffmann GF, Charpentier C, Mayatepek E et al: Clinical and biochemical phenotype in 11 patients with mevalonic aciduria. Pediatrics 1993; 91: 915-921. 
8 Houten SM, Frenkel J, Waterham HR: Isoprenoid biosynthesis in hereditary periodic fever syndromes and inflammation. Cell Mol Life Sci 2003; 60: 1118-1134.

9 Touitou I, Lesage S, McDermott M et al: INFEVERS: an evolving mutation database for auto-inflammatory syndromes. Hum Mutat 2004; 24: 194-198.

10 Cuisset L, Drenth JP, Simon A et al: Molecular analysis of MVK mutations and enzymatic activity in hyper-IgD and periodic fever syndrome. Eur J Hum Genet 2001; 9: 260-266.

11 Houten SM, Koster J, Romeijn GJ et al: Organization of the mevalonate kinase (MVK) gene and identification of novel mutations causing mevalonic aciduria and hyperimmunoglobulinaemia $\mathrm{D}$ and periodic fever syndrome. Eur J Hum Genet 2001; 9: 253-259.

12 Frenkel J, Kuis W: Overt and occult rheumatic diseases: the child with chronic fever. Best Pract Res Clin Rheumatol 2002; 16: $443-469$.

13 Houten SM, van Woerden CS, Wijburg FA, Wandres RJ, Waterham HR: Carrier frequency of the V377I (1129G $>$ A) MVK mutation, associated with Hyper-IgD and periodic fever syndrome, in the Netherlands. Eur J Hum Genet 2003; 11: 196-200.

14 Saulsbury FT: Hyperimmunoglobulinemia D and periodic fever syndrome (HIDS) in a child with normal serum IgD, but increased serum IgA concentration. J Pediatr 2003; 143: 127-129.

15 Di Rocco M, Caruso U, Waterham HR, Picco P, Loy A, Wanders RJ: Mevalonate kinase deficiency in a child with periodic fever and without hyperimmunoglobulinaemia D. J Inherit Metab Dis 2001; 24: $411-412$.

16 Picco P, Gattorno M, Di Rocco M, Buoncompagni A: Nonsteroidal anti-inflammatory drugs in the treatment of hyper-IgD syndrome. Ann Rheum Dis 2001; 60: 904.
17 Obici L, Manno C, Onetti Muda A et al: First report of systemic reactive (AA) amyloidosis in a patient with the hyperimmunoglobulinemia D with periodic fever syndrome. Arthritis Rheum 2004; 50: 2966-2969.

18 Ostuni P, Vertolli U, Marson P: Atypical hypergammaglobulinaemia D syndrome with amyloidosis: an overlap with familial Mediterranean fever? Clin Rheumatol 1996; 15: 610-612.

19 Stojanov S, Lohse P, Lohse P et al: Molecular analysis of the MVK and TNFRSF1A genes in patients with a clinical presentation typical of the hyperimmunoglobulinemia $\mathrm{D}$ with periodic fever syndrome: a low-penetrance TNFRSF1A variant in a heterozygous MVK carrier possibly influences the phenotype of hyperimmunoglobulinemia $\mathrm{D}$ with periodic fever syndrome or vice versa. Arthritis Rheum 2004; 50: 1951-1958.

20 Houten SM, Romeijn GJ, Koster J et al: Identification and characterization of three novel missense mutations in mevalonate kinase cDNA causing mevalonic aciduria, a disorder of isoprene biosynthesis. Hum Mol Genet 1999; 8: $1523-1528$.

21 Simon A, Mariman EC, van der Meer JW, Drenth JP: A founder effect in the hyperimmunoglobulinemia $\mathrm{D}$ and periodic fever syndrome. Am J Med 2003; 114: 148-152.

22 Raupp P, Varady E, Duran M, Wanders RJ, Waterham HR, Houten SM: Novel genotype of mevalonic aciduria with fatalities in premature siblings. Arch Dis Child Fetal Neonatal 2004; 89: F90-F91.

$23 \mathrm{Fu} \mathrm{Z}$, Fu Z, Wang M, Potter D, Miziorko HM, Kim JJ: The structure of a binary complex between a mammalian mevalonate kinase and ATP: insights into the reaction mechanism and human inherited disease. J Biol Chem 2002; 277: 18134-18142. 